\title{
RECENT STUDIES OF THE NORTH MAGNETIC DIP POLE*
}

\author{
K. Whitham, E. I. Loomer, and E. Dawson $\dagger$
}

\section{Introduction}

\begin{abstract}
The Division of Geomagnetism of the Dominion Observatory is often approached for information on the north magnetic dip pole and its motions by cartographers, students of geomagnetism and aeronomy, and individuals or institutions interested in the Arctic. The authors have recently re-examined the relevant scientific data available to them in order to provide the best possible estimate of the secular motion of the dip pole, and consequently to determine its position for the world declination charts, epoch 1960.0. The opportunity is taken to comment on the geophysical significance of the dip pole and its secular motion.
\end{abstract}

\section{Pole determinations prior to 1950}

Madill (1948) has described determinations of the position of the north magnetic dip pole by Ross and Amundsen, and outlined some of the results of the post-war arctic surveys carried out by this Division. The successive surveys, "Operations Magnetic" made in co-operation with the R.C.A.F., were discontinued after the 1950 field season. Hutchison derived the mean position of the pole for epoch 1950.0 in excellent unpublished work on the reduction of the results from these arctic field stations. To do this he constructed a chart of the two horizontal components of the earth's field, obtained by resolving the total horizontal intensity parallel and perpendicular to the Greenwich grid direction at any point (see Appendix). The two components $\mathrm{U}$ (grid-north component) and $\mathrm{V}$ (grid-east component) were plotted on polar stereographic projection and contoured graphically, using for interpolation and adjustment the orthogonal relation $\mathrm{dU} / \mathrm{dv}=$ $\mathrm{dV} / \mathrm{du}$, which holds approximately at high latitudes. Small $\mathrm{u}, \mathrm{v}$ are parameters of position. This relationship can be derived from Maxwell's equations by assuming that the contributions to the surface field from sources external to the earth are negligible (Hutchison 1949). The pole is located at the intersection of the $\mathrm{U}=\mathrm{O}$ and $\mathrm{V}=\mathrm{O}$ contours. The reduced data from surveys carried out prior to 1950 by the Dominion Observatory give

* Published with the permission of the Deputy Minister, Department of Mines and Technical Surveys, Ottawa, Canada.

$\dagger$ Dominion Observatory, Ottawa. 
a somewhat indefinite intersection point, and the best estimate of the position for epoch 1950.0 was $74^{\circ} \mathrm{N}$., $100^{\circ} \mathrm{W}$. This position was derived after correcting the field station data for diurnal variation and the data from different years for secular variation. However, the presence of undetected crustal anomalies in the earth's magnetic field and the unknown annual variation could make this an erroneous estimate. After examining the magnetograms of the magnetic observatory at Resolute, Cornwallis Island, only some 100 miles from the dip pole, we now know that systematic errors caused by the observations having been made mostly in July and August are probably less than about 5 miles. The effect of undetected crustal anomalies is more difficult to assess. The average density of magnetic field stations within a few hundred miles of the position of the dip pole is one station per 7,000 square miles. Using the curves published by Serson and Hannford (1957), (which refer to more southerly latitudes and show the root mean square errors in magnetic charts produced by linear interpolation between field stations), we have estimated that the uncertainty in the pole position is $\pm 1.2^{\circ}$ in longitude and $\pm 0.6^{\circ}$ in latitude. However, this result is only true in a statistical sense. It ignores the differences in geological structure between the Arctic Archipelago and other regions, and neglects the use of the orthogonality relationship mentioned above as an aid to mapping. We consider it likely that these estimates are too high, and experimental observations described below certainly suggest that near the dip pole the effect of anomalies is not too serious. Rather arbitrarily therefore, it appears reasonable to adopt uncertainties of \pm 20 miles, equivalent to $\pm 0.3^{\circ}$ in latitude and $\pm 1.2^{\circ}$ in longitude, for the 1950.0 epochal position.

In 1945 a large amount of compass data was obtained from the flights of the British Lancaster "Aries" (Maclure 1946) on tracks near Amundsen's pole position, and on a flight from Whitehorse, Y.T. to Shawbury, England. The position of the dip pole estimated from the data was near $74^{\circ} \mathrm{N}$., $100^{\circ} \mathrm{W}$. In view of the serious deviation troubles inherent in the use of compasses in a region of greatly reduced horizontal intensity, and the distance of the flight paths from the true position of the dip pole, this comparatively accurate determination represents a remarkable achievement. It also suggests that crustal anomalies cannot be very large around Boothia Peninsula and Prince of Wales Island.

\section{Pole determinations after 1950}

In 1953 the recently completed three-component airborne magnetometer of the Dominion Observatory (Serson, Mack, and Whitham 1957) was test flown over all the provinces of Canada in an R.C.A.F North Star. Two of the flights passed close to the estimated position of the dip pole: one of these was northward along the western side of Prince of Wales Island, turning easterly along Barrow Strait to Cornwallis Island and the second was westerly from Resolute to Viscount Melville Sound with a turn to the south near the mouth of M'Clintock Channel. The main purpose of these flights was to test 
the operation of the equipment under all field and flight conditions that could reasonably be expected in Canada; the determination of the dip pole position was a secondary aspect of the flight program. The magnetic declinations measured along 75 miles of flight-lines within a distance of 50 miles from the dip poles were corrected, so far as possible, for the effects of aircraft magnetism. The estimated centre of the area of convergence of the linearly extrapolated magnetic meridians was $74.4^{\circ} \mathrm{N} ., 98.1^{\circ} \mathrm{W}$. Unfortunately, errors in position and uncertainties in the corrections for aircraft magnetism and its changes are such (Serson and Whitham 1958) that the position of the dip pole determined in this way is uncertain by at least $\pm 0.2^{\circ}$ in latitude and $\pm 1.6^{\circ}$ in longitude. Three points, however, should be emphasized. Although only declination information was used to locate the dip pole, the instrument is not a compass and is not affected in the same way by reduction of the directive force near the pole. Secondly, the small area of convergence obtained again suggests that local anomalies in this region are not large. It would therefore appear to be quite reasonable to predict the approximate position of the dip pole by the convergence of magnetic meridians provided the stations used are less than about 100 miles from the dip pole. (see also Steen, Russeltvedt, and Wasserfall 1933). This is entirely different from assuming that the compass needle points toward the magnetic pole at all latitudes. Thirdly, the dip pole position given above is not corrected for magnetic disturbances, since such corrections, under the conditions existing at the time, are equivalent to less than the estimated uncertainty in the position as quoted above.

In 1955 the United States Naval Ordnance Laboratory vector airborne magnetometer was flown in the neighbourhood of the dip pole. The final results of these flights are not known to the authors, but preliminary estimates communicated privately by Stockwell in 1956 placed the dip pole in Viscount Melville Sound, north of Prince of Wales Island.

Whitham and Loomer (1956) have described the diurnal and annual motions of the north magnetic dip pole produced by disturbance variations of external origin, using the data then available from the magnetic observatory at Resolute. They also concluded that in 1955 the secular variation field of internal origin was producing a secular drift in the position of the dip pole of about 4 miles per year slightly east of north. Their general conclusions regarding the magnitude and nature of the dip pole motion produced by ionospheric and extra-terrestrial currents remain unchanged. Thus the daily track of the dip pole at a time of moderate magnetic disturbance approximates an ellipse with a long axis of ca. 30 miles running roughly north-south and a short axis of ca. 18 miles east-west. During a severe disturbance the effective position of the dip pole can easily shift some 50 to 100 miles.

The additional data now available consist of an extended series of continuous three-component magnetic records from the magnetic observatory at Resolute (50 months as compared with 20 months available in 1956), and a new series of isomagnetic charts for Canada for epoch 1955.0, prepared on 
a scale of 100 miles to the inch by Madill and Dawson (1956) and Dawson (1956). These writers compiled the latter charts independently of Hutchison's earlier work, although they used the same basic data and in addition data from northern observatories, data obtained by Loomer during the cruise of the Labrador through the northwest passage in 1954, and by Serson and Whitham during arctic flights in 1953 and 1954. The data now provide a good basis for estimating the present secular drift of the dip pole, and hence, if we know the position for one epoch, for estimating that for a later one. For the reasons outlined earlier it seems reasonable to adopt the position $74^{\circ} \pm 0.3^{\circ} \mathrm{N}$., $100^{\circ} \pm 1.2^{\circ} \mathrm{W}$. at 1950.0 as the base position for a calculation of this kind.

\section{The present secular motion of the north magnetic dip pole}

Because of the proximity of the Resolute magnetic observatory to the dip pole, the secular variation of the earth's magnetic field at the two locations must be very nearly identical, if it is assumed that the secular change has its origin inside the core of the earth. Since November 1953, continuous measurements of the magnitude and direction of the earth's magnetic field have been made at Resolute. Fig. 1 shows a plot of the mean annual values of the geographic north (X) and geographic east (Y) components of the horizontal magnetic field for the years 1954 to 1957 inclusive. In addition, two points prior to 1954 are plotted in the X-plot. These were computed from single component (declination) records obtained during 1952 and 1953 , suitably corrected for the effect of changing inclination in a small ambient field. In the Y-plot, the three points shown prior to 1954 were estimated from older scattered absolute values after making approximate corrections for disturbance so far as is now possible. Least-square calculations assuming a constant secular change during this time give the slopes of the best straight lines fitting the data from 1954 to 1957 inclusive. These values were $\dot{X}=+25$ gammas per year and $\dot{\mathrm{Y}}=+4$ gammas per year ( 1 gamma $=10^{-5}$ oersted $)$. The declination results show that the rate of change of $\mathrm{X}$ must have been constant for some years prior to 1954 , and the additional $\mathrm{Y}$ data, although known to be of only secondary accuracy for this purpose, strongly suggest that the rate of change of $\mathrm{Y}$ previous to 1954 cannot have been very different from 4 gammas per year. For example, if quadratic or cubic expressions in time are fitted to the $\mathrm{Y}$ data from 1954 to 1957 , the extrapolated value of $\mathrm{Y}$ around 1950 is numerically very much larger than our estimate from corrected old field observations. We conclude that although the rate of increase of $\mathrm{Y}$ is apparently decreasing, the results at present available suggest that a mean rate of 4 gammas per year in the last decade is the most reasonable estimate.

Assuming the approximate longitude of the dip pole to be $100^{\circ} \mathrm{W}$., the rate of change of $U$ and $V$ can be calculated from $\dot{X}$ and $\dot{Y}$. (Appendix). Hutchisons's unpublished charts show the regional field gradients at the dip pole in the $U$ and $V$ components to be 8 gammas per mile and 4 gammas per mile, respectively. Therefore, if the secular change is regarded as being 
produced by the drift of the regional field, without there being any appreciable change in form near the dip pole, the secular motion of the dip pole is 5 (.6) miles per year to the north and 1(.1) miles per year to the east. This estimate is about 20 per cent greater than the earlier estimate of Whitham and Loomer (1956), which was based on much less data. The assumption made regarding comparatively minor changes in the form of the regional field near the dip pole over a half-decade or so is confirmed by the results of a similar method using the regional field gradients shown on the $\mathrm{X}, \mathrm{Y}$ isomagnetic charts of Dawson (1956) for epoch 1955.0. The X, Y contours, in contrast to the $\mathrm{U}, \mathrm{V}$ ones, show marked curvature in polar regions, making the determination of the relevant field gradients more uncertain. However, a similar process leads to a secular motion of $5(.5)$ miles per year to the north and 0.(.4) miles per year to the east. The close agreement in magnitude and direction between the secular drift values obtained by using the two sets of charts confirms the essential correctness of the assumption. This might also be expected because the change in magnitude of the axial dipole of the earth has a very small effect on the horizontal component of force at high latitudes.

A third estimate of the secular motion can be made by using potential theory and essentially the same data, although with different approximations. The lines of equal horizontal intensity display a considerably elongated pattern in the Arctic (Madill and Dawson 1956). If the eccentricity of this pattern is uniform for all isomagnetic contours of low intensity, the pole will be at the centre of the elliptical contours. In fact, however, the position of the dip pole is far south of the centre of the pattern and this has led to the suggestion that in reality there may be two poles, with a counterpart to the north magnetic dip pole that we have been discussing situated in the remotest part of the Arctic Basin. Recent Soviet observations and studies (Anon. 1954), however, apparently discredit the existence of such a second pole. Consequently, it seems worth while to neglect the fact that the dip pole is not at the centre of symmetrical elliptical H-contours. It is then possible to write the equation of the lines of equal potential in elliptical form. If the axes $\mathrm{Ox}^{\prime}, \mathrm{Oy}^{\prime}$ are taken to coincide with the principal axes of the ellipse of lowest intensity that can be determined $(\mathrm{H}=1000$ gammas $)$, and if the origin is taken to be at the dip pole, the potential $\mathrm{V}$ at $\left(\mathrm{x}^{\prime}, \mathrm{y}^{\prime}\right)$ can be written

$$
\mathrm{V}=\mathrm{V}_{0}+\frac{\mathrm{a}}{2} \mathrm{x}^{\prime 2}+\frac{\mathrm{b}}{2} \mathrm{y}^{\prime 2} \text {. }
$$

Therefore, $X^{\prime}=-\frac{\partial v}{\partial x^{\prime}}=-a x^{\prime}$

$$
\mathrm{Y}^{\prime}=-\frac{\partial \mathrm{v}}{\partial \mathrm{y}^{\prime}}=-\mathrm{by}^{\prime}
$$

and since $\mathrm{H}^{2}=\mathrm{X}^{\prime 2}+\mathrm{Y}^{\prime 2}$

$\mathrm{H}^{2}=\mathrm{a}^{2} \mathrm{x}^{\prime 2}+b^{2} \mathrm{y}^{\prime 2}$ is the equation of the isomagnetic lines in $\mathrm{H}$. The velocity of the pole parallel to $O x^{\prime}$ is $v_{x^{\prime}}=\dot{X}^{\prime} / a$ and parallel to $O y^{\prime}$ it is $v_{y^{\prime}}=\dot{\mathrm{Y}}^{\prime} / \mathrm{b}$. Using the best determination by Dawson, revised to epoch 1960.0, of the contour for $\mathrm{H}=1000$ gammas, which is shown in Fig. 2, then $\mathrm{Ox}^{\prime}$ is rotated about $13^{\circ}$ west of geographic north and $a=6$ gammas per mile, $b=9$ gammas 

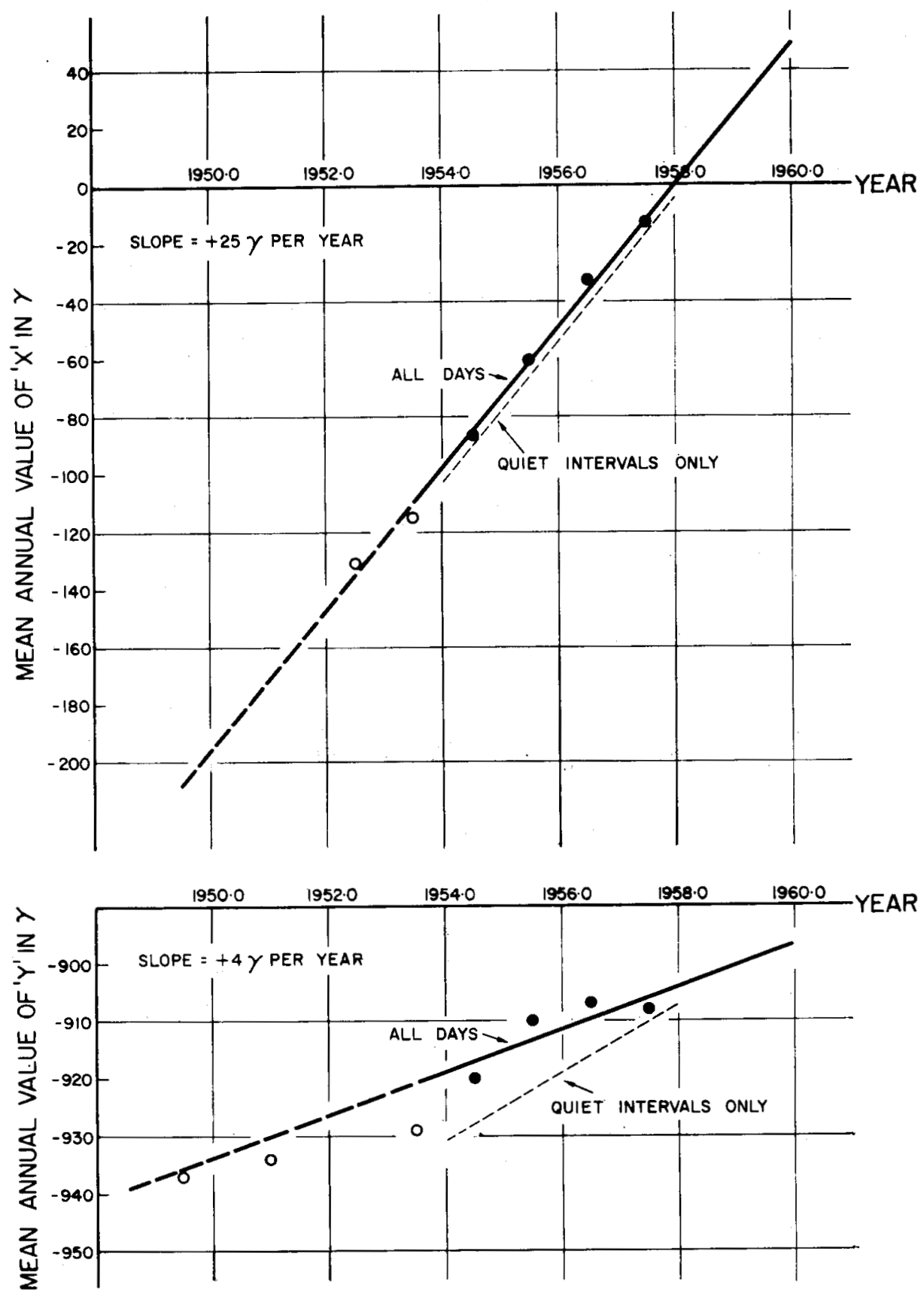

Fig. 1. The mean annual values of the geographical components of the horizontal magnetic field intensity at the magnetic observatory at Resolute for the years 1954 to 1958. 
per mile. After calculating $\dot{\mathrm{X}}^{\prime}, \dot{\mathrm{Y}}^{\prime}$ from $\dot{\mathrm{X}}, \dot{\mathrm{Y}}$, estimating $\boldsymbol{r}_{\mathrm{x}^{\prime}}, \boldsymbol{v}_{\mathrm{y}^{\prime}}$ and resolving back to geographical co-ordinates, the secular motion can be estimated to be $3(.9)$ miles per year to the north and $0(.2)$ miles per year to the east. Considering the approximation involved, these results are in substantial agreement with those given above, the mean of which indicates a secular drift of 5.5 miles per year to the north and $0(.7)$ miles per year to the east.

We conclude therefore that the magnitude and direction of the present secular drift are comparatively accurately known. One source of systematic error is that in taking mean annual values at high latitudes, the effect of ionospheric and extra-ionospheric sources may not be truly zero. There is in fact good evidence that this is so, and in Fig. 1 the dotted lines show the leastsquare slopes obtained by fitting the mean annual values of the magnetically quiet intervals only. These slopes should be substantially free of any such effect since at quiet times, by definition, disturbances are at a minimum. The selection of quiet intervals was made using the tables prepared by the International Association of Geomagnetism and Aeronomy, and by inspection. The least-square slopes obtained were $\mathrm{X}=+25$ gammas per year and $\mathrm{Y}=+6$ gammas per year. If these estimates are used in the above calculations, the secular drift is unchanged in magnitude but is very slightly more easterly. The displacement of the two sets of lines in Fig. 1 shows that the averaged effect of disturbance is not quite zero but is a vector of a few gammas directed approximately northeast (to the geomagnetic pole mentioned later) ${ }^{1}$.

\section{The estimated position of the dip pole, epoch 1960.0}

The predicted movement in this decade is $0.8^{\circ}$ in latitude and $0.4^{\circ}$ in longitude. Assuming Hutchison's 1950.0 position, the predicted position for epoch 1960.0 becomes $74.8^{\circ} \pm 0.3^{\circ} \mathrm{N} ., 99.6^{\circ} \pm 1.2^{\circ} \mathrm{W}$. We are more confident of its present secular motion than its present absolute position, particularly in longitude. This is illustrated in Fig. 3.

\section{The geophysical significance of the dip pole and its motion}

Quite naturally much scientific interest is attached to the motion of the north magnetic dip pole (Madill 1948; Beals, et al. 1954). The revised estimates outlined above are in good agreement with those made by Whitham and Loomer (1956), and the earlier conclusion that the present northerly drift substantially agrees with the mean northerly motion for the last halfcentury is confirmed. The present very small easterly motion is obviously much more uncertain, and the distribution of mean annual values shown in Fig. 1 suggests it may be decreasing. In any case, because the position of the

1 Note added in proof. Since the completion of this paper we have received information from Rear Admiral Charles Pierce (private communication) that the U.S. Coast and Geodetic Survey have computed the 1960 position of the north magnetic dip pole as $74.9^{\circ} \mathrm{N}$., $101.8^{\circ} \mathrm{W}$. It has been suggested that a compromise position $74.9^{\circ} \mathrm{N}$., $101^{\circ} \mathrm{W}$. be adopted for use on Canadian and world charts. Although this position will be to the west of the 1950 position, we are confident that the secular motion of the dip pole is slightly east of north. 


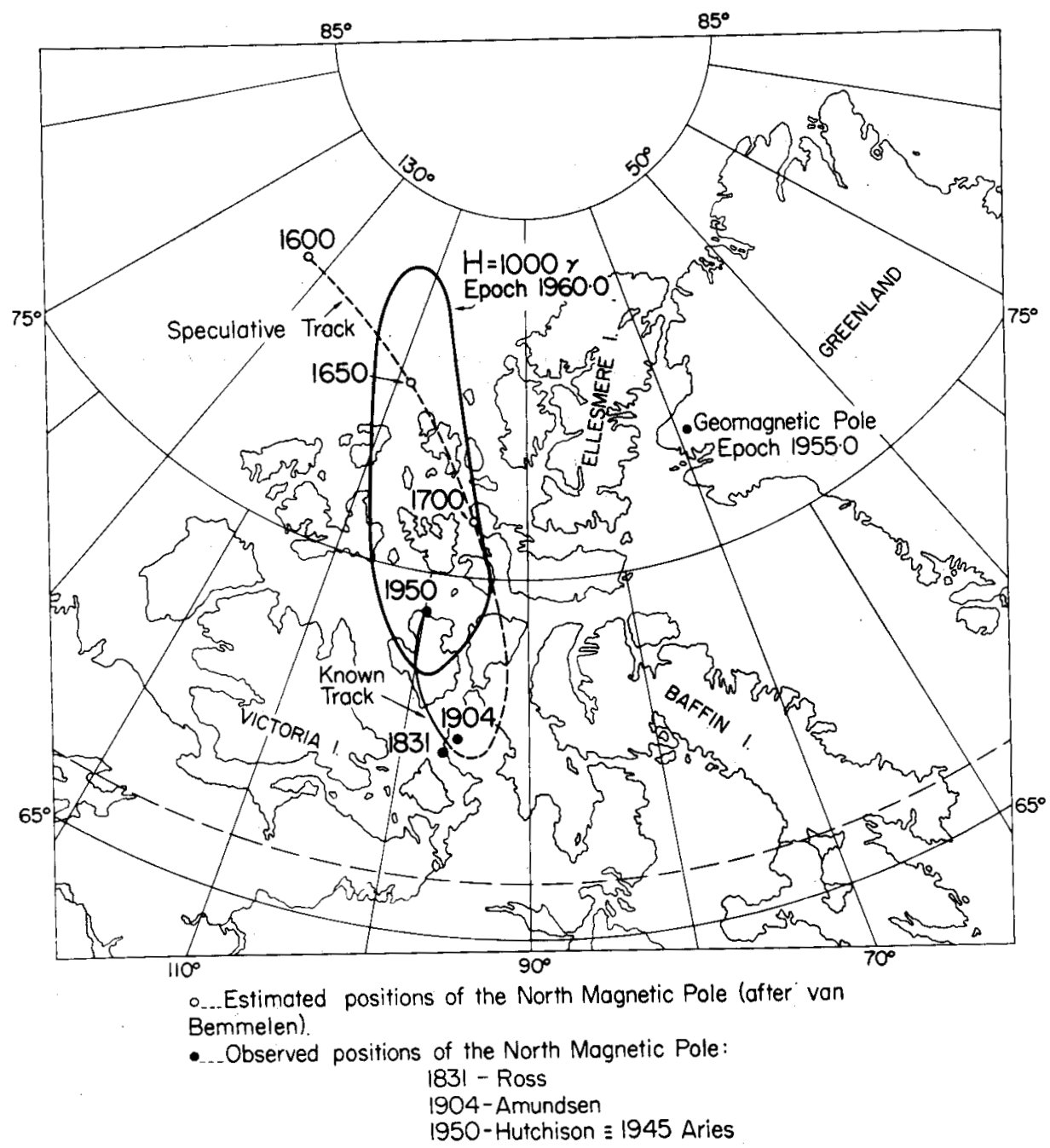

Fig. 2. The speculative and known tracks of the north magnetic dip pole and the position of the contours for $\mathrm{H}=1000$ gammas at epoch 1960.0 .

pole depends entirely on the magnitude of the higher harmonic terms in a spherical harmonic expansion of the main field, its motion is apparently not related in any simple way to the broad-scale core motions deduced by an analysis of the eccentric dipole at different epochs (Vestine 1953). We now know this result is true of the geomagnetic field as a whole in Canada (Whitham 1958). It should, of course, be clear that the secular motion of the pole is a consequence of secular variation and not its cause. Consequently, this motion can provide a simple picture of the secular variation in a region very close to the pole only.

It has been suggested that the secular motion is really a linear oscillation in which constraints imposed by a fixed magnetic anomaly (the great arctic 
anomaly) impose essentially linear trapping on what is thought to be fundamentally a westward circular motion around the geographic pole (Hope 1957a and 1957b). Much of the evidence for this rests on the reconstructed pole positions of van Bemmelen, which are shown in Fig. 2. These however are known to be unreliable since they depend on magnetic information from low latitudes only. When magnetic data from low latitudes only are available, the position of the dip pole can best be estimated by using the techniques of spherical harmonic analysis. With a finite number of terms in the expansion used (customarily 48 terms) the pole positions deduced in this way are in error by 100 miles or more, even today with our vastly increased knowledge of the distribution of the intensity of the magnetic field. Simpler methods, such as assuming dipoles or extrapolating isogons or both, lead to enormous errors. In addition, the authors consider that the elongated horizontal intensity contours can be explained by non-dipole sources inside the core, and they know of no evidence that convincingly associates the so-called "anomaly" with the geology of the area. This, however, is not denying the existence of crustal anomalies. Indeed, it would be surprising if they did not exist, and they can easily cause errors in the determination of the position of the pole.

In our opinion, therefore, the future motion of the pole cannot be reliably predicted at this stage because we cannot predict the secular variation of the earth's field. Our knowledge of the life times of isoporic foci (centres of rapid annual change of the magnetic field) does suggest that the present northward motion is likely to persist within a factor of two in magnitude for the order of 100 years. Even this cautious prediction is really only an intelligent guess. The significance and persistence of the eastward motion is more difficult to assess. Whitham (1958) has commented on the lack of evidence in Canadian data for the generally accepted westward-drift of the non-dipole component of the earth's field, and has shown that there is no evidence for a 480-year rotation period. Studies of rock magnetism have suggested that, among other phenomena, repeated reversals of the polarity of the earth's main magnetic field have occurred. The average position of the magnetic dip pole during a time of ca. 100,000 years might be expected to be that of the geographic north pole, because of the dominant influence of the Coriolis force on core motions, and reversals apparently occur about once every 10,000 years. This corresponds to a rate of change of magnetic latitude at only one fourth of the present rate, which can therefore not be considered as unusual.

The significance of the dip pole and of the elongated shape of the isomagnetic contours in studies of magnetic disturbance is still obscure. Most synoptic studies show that the axis of the dipole at the earth's centre, which best represents the earth's magnetic field and can be thought of as intersecting the surface of the earth at the so-called geomagnetic poles, is of more importance in the study of aeronomy. The northern geomagnetic pole is only very slowly changing position and its latest determined position was $78.3^{\circ} \mathrm{N}, 69^{\circ} \mathrm{W}$ at epoch 1955.0 (Finch and Leaton 1957), some 600 miles northeast of the magnetic dip pole. However, many of the details concerning 


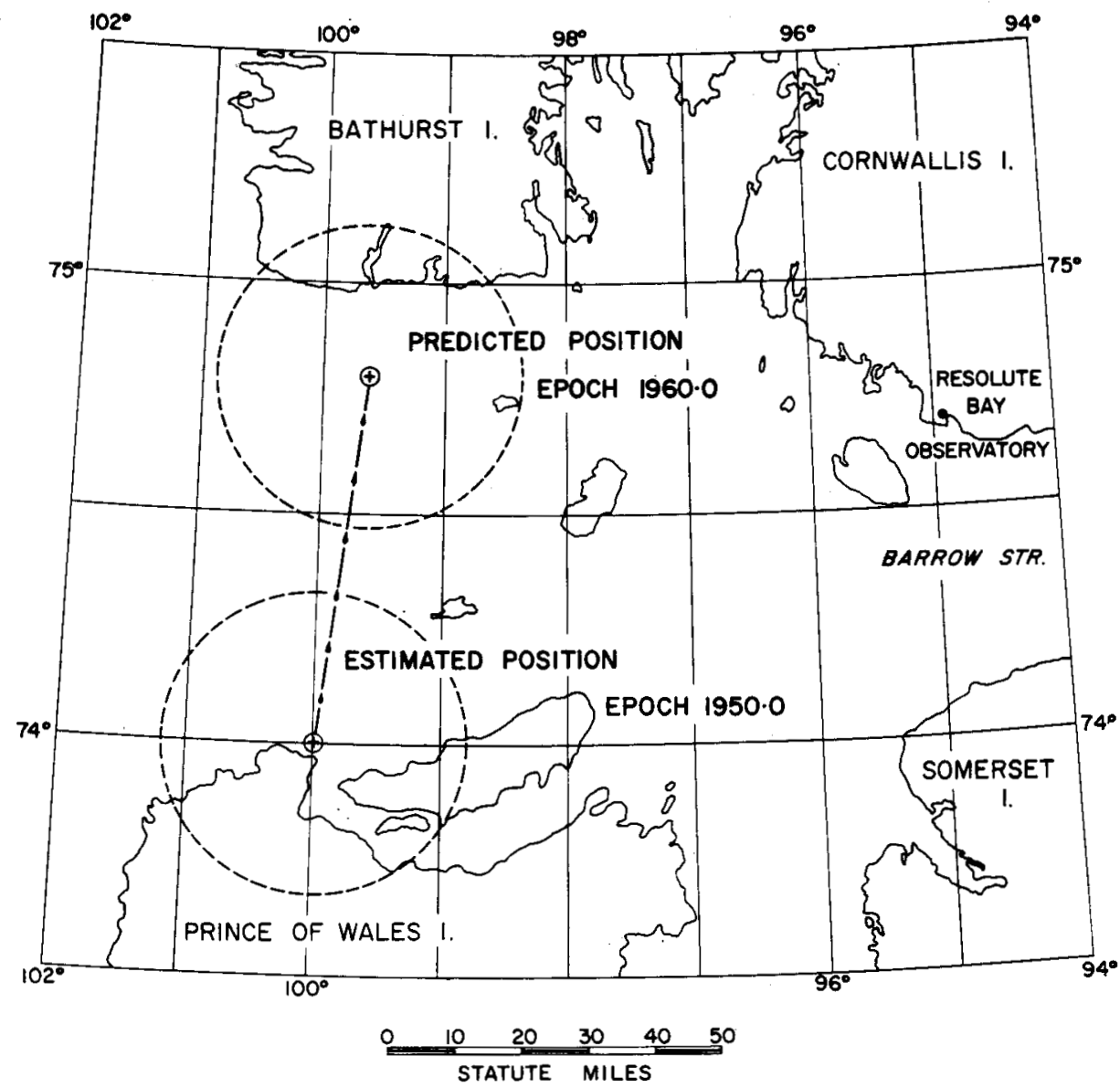

Fig. 3. The predicted dip pole position, epoch 1960.0, and the present secular motion of the dip pole. The circles indicate the estimated uncertainty of the true epochal positions.

the possible penetration of charged particles into the ionosphere along magnetic lines of force, and the interaction of the magnetic field with streams of charged particles from the sun may be partially explained by the deviation of the earth's field from that of a dipole. The position and motion of the dip pole are convenient indicators of this deviation from a dipole field.

\section{The zero isopor of declination}

This passes through the dip pole in the direction of the change of horizontal field intensity. Consequently its direction at the pole should be in the direction of the pole motion. There is a small discrepancy between the direction shown in the isogonic chart for Canada, epoch 1955.0 (Madill and Dawson 1956), and the direction of motion deduced in this paper and by Whitham and Loomer (1956). This will be corrected in the next isogonic chart for epoch 1960.0, which is in preparation. 


\section{Acknowledgements}

We wish to thank Mr. R. G. Madill and Dr. C. S. Beals for their encouragement, and for suggestions during the preparation of this manuscript.

\section{Appendix}

Greenwich grid system

On a polar stereographic projection, one draws straight lines parallel to a fixed meridian, in this case the meridian of Greenwich. Just one line

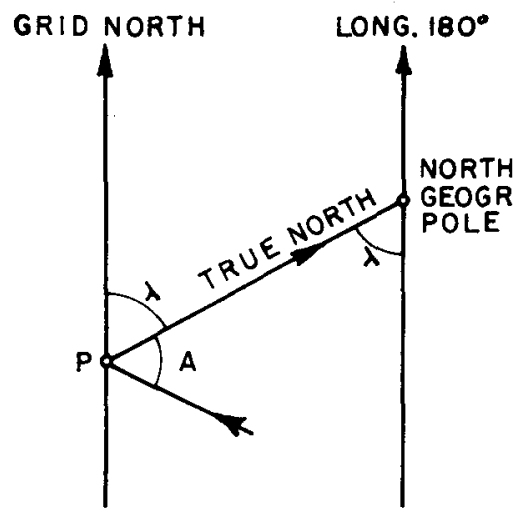
passes through any place $P$ on the map. The direction of this line through $P$ (strictly the direction parallel to the direction of the true north on the Greenwich meridian) is called grid north. A grid bearing is obtained from the corresponding true bearing by adding (if west) or subtracting (if east) the longitude of $P$.

$$
\begin{aligned}
& \text { True bearing }=\mathrm{A} \\
& \text { Longitude }=\lambda \\
& \text { Grid bearing } \mathrm{G}=\mathrm{A} \pm \lambda
\end{aligned}
$$

where $\lambda$ is positive for west longitude and negative for east longitude.

The advantage of this system is that the north geographic pole is no longer a singular point. In simplifying the problem of polar direction, the grid system also simplifies the pattern of isogonic lines for magnetic declination. There is no longer a singularity at the north geographic pole; the isogonic lines radiate from the north magnetic dip pole only.

For purposes of analysis, the earth's total magnetic vector $\bar{F}$ is usually resolved into three orthogonal components $X$ (true north component), $Y$ (true east component) and $\mathrm{Z}$ (vertical component). $\mathrm{X}$ and $\mathrm{Y}$ are generally computed from the measured values of $\mathrm{D}$ (magnetic declination) and $\mathrm{H}$ (the horizontal component of $\overline{\mathrm{F}}$ )

$$
\begin{aligned}
& \mathrm{X}=\mathrm{H} \cos \mathrm{D} \\
& \mathrm{Y}=\mathrm{H} \sin \mathrm{D}
\end{aligned}
$$

This leads to geometrical complications in $\mathrm{X}$ and $\mathrm{Y}$ at the north geographic pole, but these complications can be eliminated by using the grid reference system. In this system we define the following terms

grid variation (grivation) $\mathrm{G}=\mathrm{D}-\lambda$

where $\lambda$ is the east longitude,

$\mathrm{D}$ is positive for east declination

and negative for west declination

$\mathrm{U}$ (grid-north component) $=\mathrm{H} \cos \mathrm{G}$

$\mathrm{V}$ (grid-east component) $=\mathrm{H} \sin \mathrm{G}$

From equations (2) (3) (4) (5) (6), the relationship between U, V, X, Y, can be expressed as follows 


$$
\begin{aligned}
\mathrm{U} & =\mathrm{H} \cos (\mathrm{D}-\lambda) \\
& =\mathrm{X} \cos \lambda+\mathrm{Y} \sin \\
\mathrm{V} & =\mathrm{H} \sin (\mathrm{D}-\lambda) \\
& =-\mathrm{X} \sin \lambda+\mathrm{Y} \cos \lambda
\end{aligned}
$$

From (7) (8), the rate of change of $U$ and $V$ is

$$
\begin{aligned}
& \mathrm{dU} / \mathrm{dt}=\dot{\mathrm{U}}=\dot{\mathrm{X}} \cos \lambda+\mathrm{Y} \sin \lambda \\
& \mathrm{dV} / \mathrm{dt}=\dot{\mathrm{V}}=-\dot{\mathrm{X}} \sin \lambda+\mathrm{Y} \cos \lambda
\end{aligned}
$$

For a more complete description of the Greenwich grid system see references (Maclure 1946) and (Hutchison 1949).

\section{References}

Anonymous, 1954. On new Soviet research and discoveries in the central Arctic. Izv. Akad. Nauk. S.S.S.R., Ser. Geog. No. 5, pp. 3-16. (English translation by E. R. Hope in T165R of Defence Research Board, Canada).

Beals, C. S., J. H. Hodgson, M. J. S. Innes and R. G. Madill, 1954. Problems of geophysics in the Canadian Arctic. Arctic 7:176-87.

Bemmelen, W. van. 1900. Die Säkular-Verlegung der magnetischen Axe der Erde. Mag. Met. Obs. 22, Batavia.

Dawson, E. 1956. Magnetic charts of Canada, epoch 1955.0 for X, Y. (Unpublished).

Finch, H. F. and B. R. Leaton. 1957. The earth's main magnetic field, epoch 1955.0 Mon. Not. Roy. Astron. Soc. Geophys. Suppl. 7:3.4-17.

Hope, E. R. 1957a. Linear secular oscillation of the northern magnetic pole. J. Geophys. Res. 62:19-27.

1957b. Rotation, pulse-disturbance and drift in the geomagnetic secular variation. J. Geophys. Res. 62: 29-42.

Hutchison, R. D. 1949. The horizontal geomagnetic field in the Canadian Arctic. Unpublished B.A.Sc. Thesis, Univ. Toronto.

1950. Magnetic chart of northern Canada, epoch 1950.0, for the Greenwich grid components of the horizontal field intensity. (Unpublished).

Maclure, K. C. 1946. Technical aspects of the 'Aries' flights. Geog. J. 107:105-25.

Madill, R. G. 1948. The search for the north magnetic pole. Arctic 1:8-18.

Madill, R. G. and E. Dawson. 1956. Magnetic charts of Canada, epoch 1955.0 for D, I, H, $Z$, and F. Dept. Mines and Tech. Surveys, Ottawa.

Schonstedt, E. O. and H. R. Irons. 1955. The N. O. L. vector airborne magnetometer, Type 2A. Trans. Am. Geophys. Union. 36:25-41.

Serson, P. H. and W. L. W. Hannaford. 1957. A statistical analysis of magnetic profiles. J. Geophys. Res. 62:1-18.

Serson, P. H., S. Z. Mack, and K. Whitham. 1957. A three-component airborne magnetometer. Pub. Dom. Obs. 29: 15-97.

Serson, P. H. and K. Whitham. 1958. A three-component airborne magnetometer. Hbuch. der Physik, Vol. 49, Berlin. Springer-Verlag. (in press).

Steen, A. S., N. Russeltvedt, and K. F. Wasserfall. 1933. The Scientific results of the Norwegian arctic expedition in the Gjoa, 1903-6. Part II Terrestrial magnetism. Geofys. Publik. Vol. 7, Oslo.

Vestine, E. H. 1953. On variations of the geomagnetic field, fluid motions and the rate of the earth's rotation. J. Geophys. Res. 58:127-45.

Whitham, K, and E. I. Loomer. 1956. The diurnal and annual motions of the north magnetic dip pole. J. Atmosph. Terr. Phys. 8:349-51.

Whitham, K. 1958. The relationships between the secular change and the non-dipole fields. Can. J. Phys. 36:1372. 